\title{
Predicting pubic arch interference in permanent prostate brachytherapy based on the specific parameters derived from nuclear magnetic resonance imaging
}

\author{
Yupeng Zheng, MD, Jixiang Wu, MD, Shan Chen, MD, Yuexin Liu, MD, Guangyin Zhang, MD \\ Department of Urology, Capital Medical University affiliated Beijing Tongren Hospital No I, Dong Jiao Min Xiang, Dongcheng District, Beijing \\ China
}

\begin{abstract}
Purpose: The aim of this study was to establish a more reliable method to predict pubic arch interference (PAI) before permanent prostate brachytherapy.

Material and methods: We retrospectively analyzed the nuclear magnetic resonance imaging (MRI) results of forty patients with prostate cancer, who were treated with permanent implantation of ${ }^{125}$ I seeds (permanent brachytherapy). We measured and calculated six parameters based on the MRI results: 1 . The prostate volume (PV); 2 . The angle of the pubic arch (AoPA); 3. The angle of PAI (AoPAI, pubic symphysis level); 4 . The height of PAI (hPAI, pubic symphysis level); 5. The maximum angle of PAI (AoPAI $\left.{ }^{\mathrm{Max}}\right) ; 6$. The maximum height of PAI (hPAI $\left.{ }^{\mathrm{Max}}\right)$. We then tested which parameters could accurately predict PAI through receiver operating characteristic (ROC) curve analysis.

Results: The results of this study demonstrated that AoPAI, hPAI, hPAI ${ }^{\mathrm{Max}}$, and AoPAI ${ }^{\mathrm{Max}}$ could predict PAI. Out of forty cases in our research, 10 cases were with PAI and 30 cases without PAI during the operation. The areas under the ROC curve for PV, AoPA, AoPAI (pubic symphysis level), hPAI (pubic symphysis level), AoPAI ${ }^{\text {Max }}$, and hPAI ${ }^{\text {Max }}$ were $0.592,0.567,0.957,0.940,0.927$, and 0.877 , respectively. The AoPAI (pubic symphysis level), hPAI (pubic symphysis level), AoPAI ${ }^{\mathrm{Max}}$, and $\mathrm{hPAI}^{\mathrm{Max}}$ were statistically correlated with PAI. The boundary values were $26.32^{\circ}, 1.13 \mathrm{~cm}$, $28.37^{\circ}$, and $1.51 \mathrm{~cm}$, respectively.

Conclusions: This new method derived from MRI has predictive value, as AoPAI, hPAI, hPAI ${ }^{\mathrm{Max}}$, and AoPAI ${ }^{\mathrm{Max}}$ could predict PAI. Taking other factors into consideration, we suggest the use of AoPAI as a novel and very reliable predictor of PAI.
\end{abstract}

J Contemp Brachytherapy 2018; 10, 5: 405-410 DOI: https://doi.org/10.5114/jcb.2018.79247

Key words: brachytherapy, nuclear magnetic resonance imaging (MRI), prostate cancer, pubic arch interference (PAI), seed implantation.

\section{Purpose}

Brachytherapy is a type of radiotherapy, in which the encapsulated radiation source(s) is (are) placed inside or next to the area to be treated. On the other hand, external beam radiotherapy uses high-energy $X$-rays (or $\gamma$-rays from a radioisotope like cobalt-60) to destroy tumors from outside of the body.

Brachytherapy has been widely used for about 30 years and is thought to be one of the most efficient treatments for prostate cancer [1]. Its efficacy is generally similar to that of radical prostatectomy [2,3], even in the era of robot-assisted laparoscopy. In the past 20 years, the emergence of three-dimensional treatment planning systems has further improved the efficacy of brachytherapy.

According to the guidelines of the American Brachytherapy Society and the European Urological Association, prostate cancer patients with prostate-specific antigen levels less than $10 \mathrm{ng} / \mathrm{l}$, tumor, lymph node, metastasis (TNM) staging less than $\mathrm{T}_{2 \mathrm{a}} \mathrm{N}_{0} \mathrm{M}_{0}$, and a Gleason score between $\leq 6$ are suitable for brachytherapy treatment without other therapy such as external beam radiotherapy or endocrine therapy. A prostate volume (PV) $>60 \mathrm{ml}$ is a contraindication for prostate brachytherapy. One challenging clinical issue for brachytherapy is pubic arch interference (PAI), which is often a limiting factor in adequate prostate coverage during transperineal brachytherapy. However, cur-

Address for correspondence: Jixiang Wu, MD, Department of Urology, Capital Medical University affiliated Received: 26.07.2018 Beijing Tongren Hospital No 1, Dong Jiao Min Xiang, Dongcheng District, No 1, Dong Jiao Min Xiang, Accepted: 25.09 .2018 Dong Cheng District, 100730 Beijing, China, phone: +86 13167369200, 凶 e-mail: 13167369200@163.com Published: 30.10 .2018 
rently, there are no reliable parameters that can consistently predict PAI, although PV $>60 \mathrm{ml}$ is generally used as a predictor of PAI. Bellon et al. [4] have reported that patients with a large PV generally have severe PAI, but the degree of interference is only loosely correlated to the PV; therefore, they concluded that the PV cannot reliably predict PAI.

Our long-term observations in clinical practice also suggest that PAI seems to be associated with a variety of different factors, and that PV $>60 \mathrm{ml}$ is in fact not a good predictor of PAI. Serious problems will occur if the PV is the only factor applied to predict PAI. Occasionally, PAI still occurs and some of the seeds cannot be implanted into the prostate, even if the PV is less than $60 \mathrm{ml}$. In this case, if the patients undergo brachytherapy, the effect of brachytherapy will be affected. In the worst scenario, the patients may not receive curative treatment at the optimal time. On the other hand, patients with a higher PV (PV > $60 \mathrm{ml})$ often have to receive neo-adjuvant endocrine therapy to reduce the PV before they can receive brachytherapy treatment. Unfortunately, neo-adjuvant endocrine therapy usually takes 3 months and has many side effects [5]. Nevertheless, those patients with PV $>60 \mathrm{ml}$ may not actually have PAI. Therefore, we need a reliable method to predict PAI. There are several methods that can be used to predict PAI, such as transrectal ultrasonography (TRUS) [6], computed tomography (CT) [4], and TRUS-CT fusion imaging [7]. However, predicting PAI by nuclear magnetic resonance imaging (MRI) has many obvious advantages. First, ultrasound imaging cannot clearly show the pubic bone. Ultrasound measurements are more likely to be subjectively influenced by the examiner (the results

Table 1. General characteristics of the patients included in this study

No. of patients (\%)

\begin{tabular}{lc}
\hline PSA $(\mathrm{ng} / \mathrm{ml})^{\star}$ & $12(30)$ \\
\hline$<10$ & $19(47.5)$ \\
\hline$>20$ & $9(22.5)$ \\
\hline Gleason score & $10(25)$ \\
\hline $2-6$ & $21(52.5 \%)$ \\
\hline $8-10$ & $9(22.5)$ \\
\hline TNM stage & $11(27.5)$ \\
\hline T1c-T2a & $26(65)$ \\
\hline T2b-T2c & $3(7.5)$ \\
\hline T3 & $20(50 \%)$ \\
\hline PV (ml) & $20(50 \%)$ \\
\hline $40-60$ & \\
\hline$>60$ &
\end{tabular}

PSA - prostate-specific antigen; TNM - tumor, lymph node, metastasis; PV prostate volume; ${ }^{*}$ Median $=15.45 \mathrm{ng} / \mathrm{ml}($ mean $=15.85$, range: $4.9-39.1 \mathrm{ng} / \mathrm{ml})$ may vary greatly from doctor to doctor). Although CT can clearly show the bony structure, the outline of the prostate is not clear. In contrast, MRI can clearly show both the pubis and prostate outline. In China, MRI is a routine examination for patients with prostate cancer; therefore, using MRI to predict PAI does not increase the patient's cost.

To further clarify this issue and to establish reliable predictors of PAI, we retrospectively derived six different parameters based on the MRI and additionally studied the predictive values of these parameters.

\section{Material and methods}

\section{Summary of the patient data}

We retrospectively reviewed 40 patients with prostate cancer, who underwent brachytherapy in our hospital from December 2010 to December 2016 (Table 1). The inclusion criteria were as follows: 1 . All cases have completed clinical date and MRI data; 2 . PV $>40 \mathrm{ml}$.

\section{Parameters derived from the MRI}

All patients underwent MRI (GE Healthcare, Milwaukee, WI, USA) before biopsy and seed implantation therapy. We measured the following six parameters based on the MRI (we consider MRI T2 weighted imaging better than T1) (Figure 1): $1 . \mathrm{PV}=\mathrm{L} \times \mathrm{W} \times \mathrm{H} \times \pi / 6$, where $\mathrm{L}$ is the length, $\mathrm{W}$ is the width, and $\mathrm{H}$ is the height of the prostate in $\mathrm{cm}^{3} ; 2$. The AoPA is the angle of the ramus ossis pubis on the MRI (not the same as the anatomical angulus subpubicus) (Figure 1A); 3. The AoPAI (pubic symphysis level) is the angle of line A and line $\mathrm{B}$ on sagittal images through the pubic symphysis (line A is the horizontal line on the posterior border of the pubic symphysis (patients in supine position), and line $\mathrm{B}$ is the line between the highest point of the anterior prostatic edge and the posterior border of the pubic symphysis) (Figure 1B); 4 . The hPAI (pubic symphysis level) is the vertical height of the highest point of the anterior prostatic edge to the horizontal line of the posterior border of the pubic symphysis $(\mathrm{cm})$ (Figure 1B); 5. The AoPAI ${ }^{\mathrm{Max}}$ is the maximum angle of line $\mathrm{A}$ and line $\mathrm{B}$ on all sagittal planes of the MRI. Line A is the horizontal line on the posterior border of the pubic arch (patients in supine position). Line B is the line between the highest point of the anterior prostatic edge and the posterior border of the pubic arch (Figure 1C); 6. The hPAI ${ }^{\text {Max }}$ is the vertical height of the highest point of the anterior prostatic edge to the horizontal line of the posterior border on the same sagittal plane of the AoPAI ${ }^{\mathrm{Max}}$ (Figure 1C).

These parameters can be conveniently measured in the MRI PACS (picture archiving and communication system).

\section{Brachytherapy procedure}

Brachytherapy equipment: prostate cancer brachytherapy therapy planning system (Flying Tenor Inc., China), the Accu2 seed locator (transrectal ultrasound and the probe: BK, USA), Mick applicator and template (Mick Radionuclear Instruments, Bronx, NY, USA). Our implant technique was adapted from the American Brachytherapy 

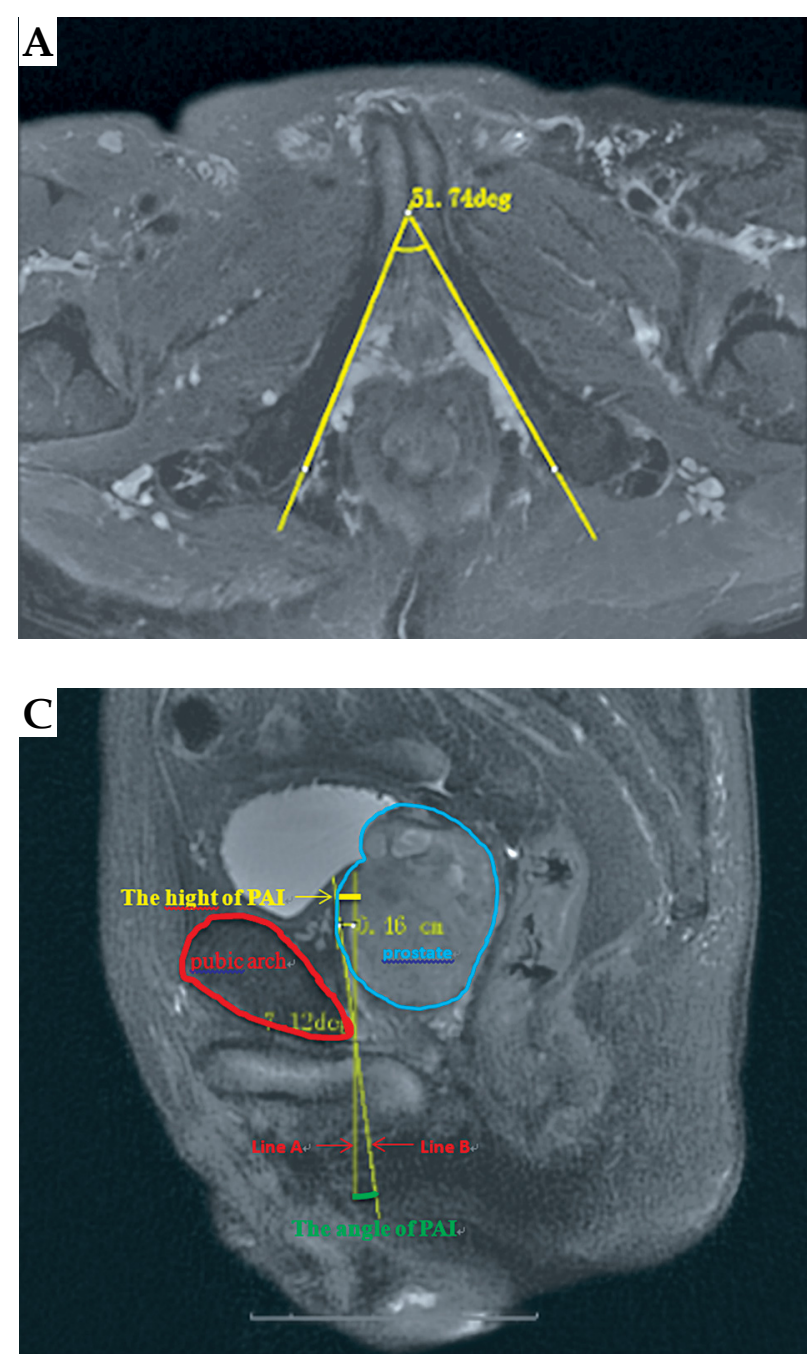

Society (ABS) [8]. Patients were positioned in exaggerated dorsal lithotomy $\left(90^{\circ}\right.$ of knee flexion, $100^{\circ}$ of hip joint flexion, and $90^{\circ}$ external rotation), head-low position by $20^{\circ}$, and the ultrasonic probe was in horizontal position. The brachytherapy plan was generated using Flying TPS (Flying Tenor Inc., China). We used $0.4 \mathrm{mCi}$ loose I-125 seeds, and the prescribed dose was $144 \mathrm{~Gy}$. When PAI occurred, we tried to increase the angle of head-low position. Unfortunately, sometimes, increasing angle has no effect because the ultrasound probe is in the rectum, and the angle of head lower could not be too much; otherwise, the ultrasound probe could not be close to the prostate, resulting in unclear ultrasound image. There were two ways to overcome it: 1) using an oblique needle with template implantation, and 2) using an oblique needle without a template. Ryu et al. [9] have reported similar method to overcome PAI. In case of PAI, multiple punctures might be required to deliver the applicator to a predetermined location to ensure the efficacy of brachytherapy.

\section{Statistical methods}

All statistical analyses were conducted by SAS software, version 9.4. For each parameter, the area under

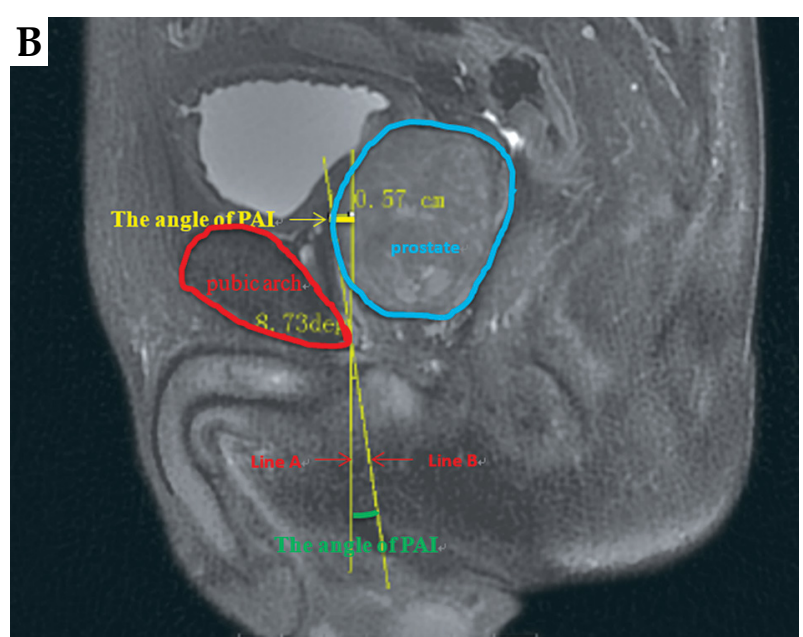

Fig. 1. A) The angle of the pubic arch (AoPA) is the angle of the rami ossis pubis on MRI, but it is not the same as the anatomical angulus subpubicus (AoPA is $51.74^{\circ}$ on this picture). B) The angle of PAI (pubic symphysis level): the angle of line A and line B on the sagittal plane through the pubic symphysis. Height of PAI (pubic symphysis level): the vertical height of the highest point of the anterior prostatic edge to line A $(\mathrm{cm})$. Line A: the horizontal line on the posterior border of the pubic symphysis (patients in the supine position). Line B: the line between the highest point of the anterior prostatic edge and the posterior border of the pubic symphysis (the angle of PAI is $8.73^{\circ}$, and the height of $\mathrm{PAI}$ is $0.57 \mathrm{~cm}$ on this picture). C) The angle of PAI (Max): the max angle of line A and line B on each sagittal plane of MRI. Height of PAI (Max): the highest point of the anterior prostatic edge to line A $(\mathrm{cm})$. Line A: the horizontal line on the posterior border of the pubic arch (patients in the supine position). Line B: the line between the highest point of the anterior prostatic edge and the posterior border of the pubic arch (the angle of PAI (Max) is $7.12^{\circ}$, and the height of PAI (Max) is $0.16 \mathrm{~cm}$ on this picture)

the receiver operating characteristic (ROC) curve was used to evaluate the predictive ability for PAI, and the Mann-Whitney method was applied to analyze the statistical difference between the areas of each parameter and the reference area of 0.5 . It was considered statistically significant if the $95 \%$ confidence interval $(\mathrm{CI})$ of the area failed to cover 0.5 .

\section{Results}

\section{PAI in brachytherapy treatment}

Among the 40 reviewed patients, ten had PAI (PV $\leq 60 \mathrm{ml}$ in 6 patients and $\mathrm{PV}>60 \mathrm{ml}$ in 4 patients). To overcome PAI, the intraoperative oblique needle method was used in nine cases, and the oblique needle method without a template plan was used in one case.

\section{Descriptive of the parameters}

Mean prostate volume was $63.76 \pm 18.36 \mathrm{ml}$, mean AoPA was $59.35 \pm 5.57$, mean AoPAI (pubic symphysis level) $21.37 \pm 7.56 \mathrm{ml}$, mean hPAI (pubic symphysis level) was $0.99 \pm 0.42 \mathrm{~cm}$, mean AoPAIMax $22.96 \pm 7.81$, and mean hPAIMax was $1.09 \pm 0.40 \mathrm{~cm}$ 
A

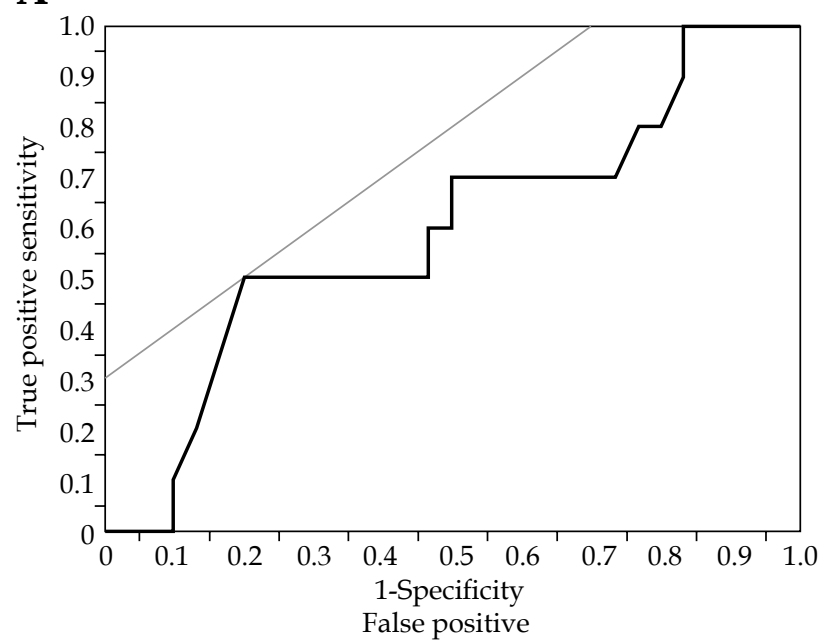

C

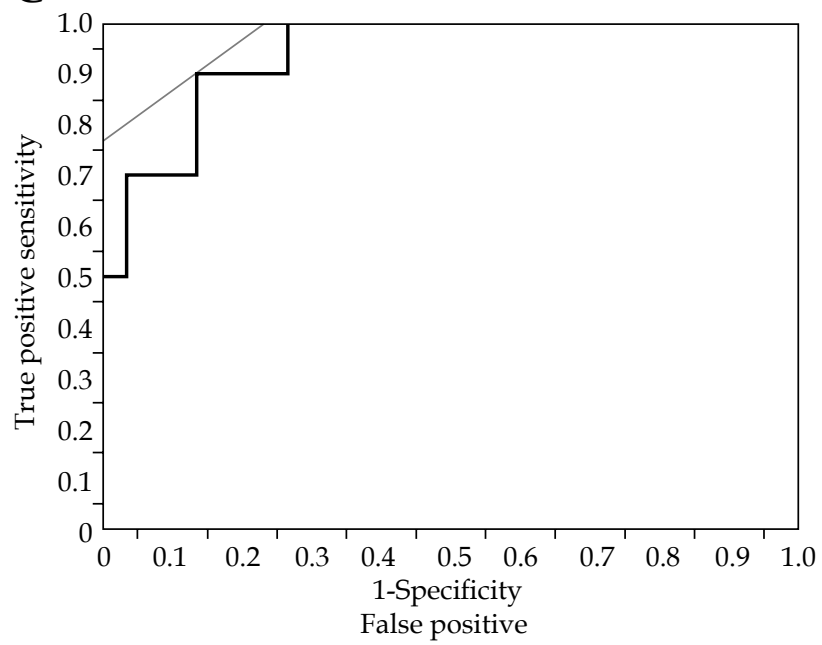

E

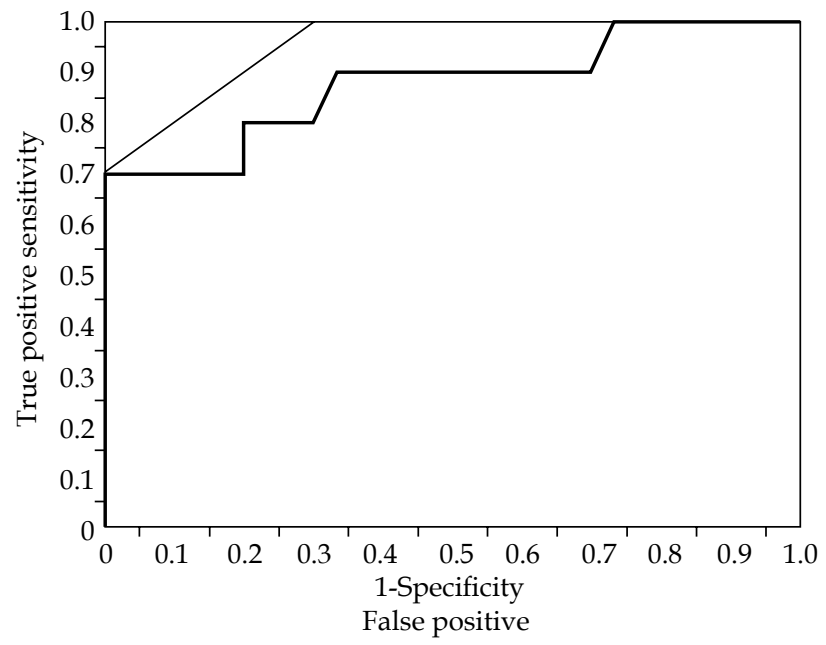

B

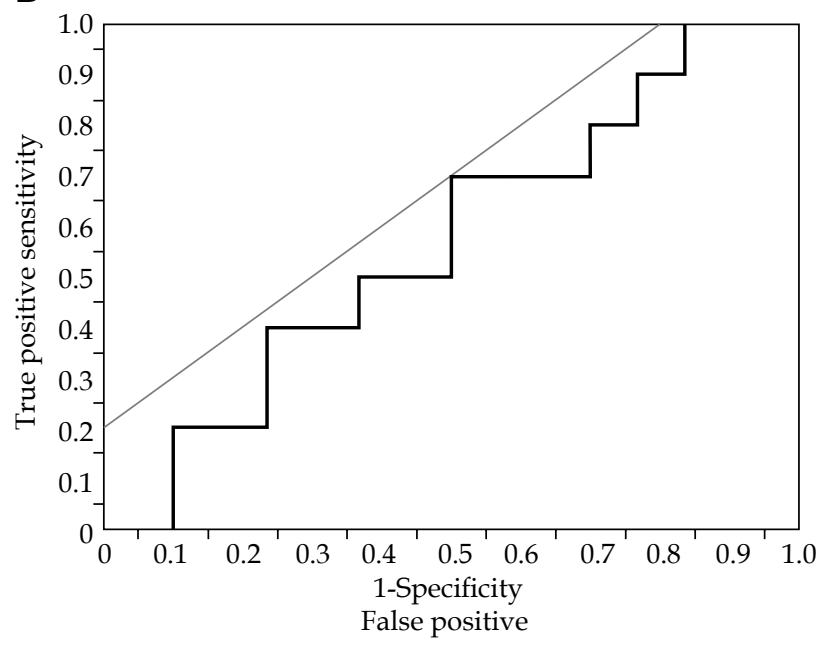

D

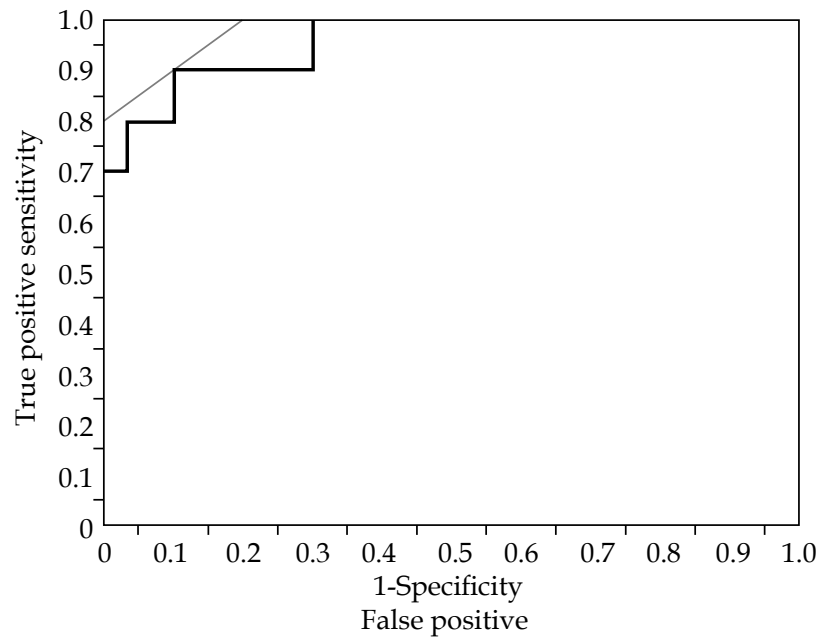

$\mathbf{F}$

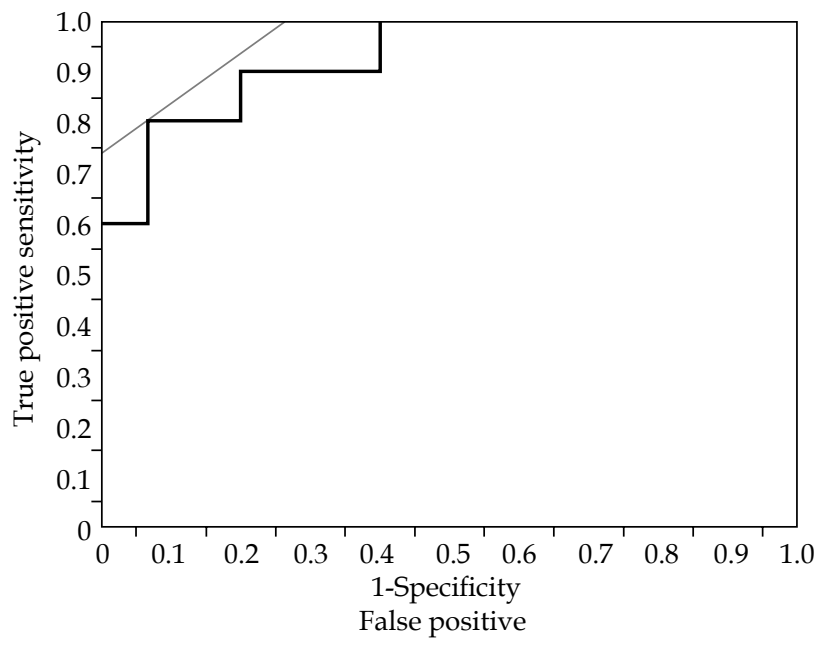

Fig. 2. A) The ROC curve of the PV and PAI suggests that PV has no predictive value for PAI $(p>0.05)$. B) The ROC curve of the AoPA and PAI suggests that AoPA has no predictive value for PAI $(p>0.05)$. C) The ROC curve of the hPAI and PAI suggests that hPAI has a significant predictive value for PAI $(p<0.05)$. D) The ROC curve of the AoPAI and PAI suggests that AoPAI has significant predictive value for PAI $(p<0.05)$. E) The ROC curve of the hPAI ${ }^{\text {Max }}$ and PAI suggests that hPAI ${ }^{\text {Max }}$ has a significant predictive value for PAI $(p<0.05)$. F) The ROC curve of the AoPAI ${ }^{\mathrm{Max}}$ and PAI suggests that AoPAI ${ }^{\mathrm{Max}}$ has significant predictive value for PAI $(p<0.05)$ 


\section{Predictive value of PV for PAI}

We used ROC curve analysis to clarify the predictive value of each individual parameter for PAI (Figure 2A). ROC curve analysis provides two bits of relevant information for each threshold. In the ROC model, the area under the curve of PV and PAI was 0.59167, the standard error was 0.110 , and the $95 \%$ CI was $0.376-0.807$. Therefore, this model indicated that PV had no significant predictive value for PAI $(p=0.2975)$. Therefore, in our research, the predictive value of PV was poor.

\section{Predictive value of AoPA for PAI}

Similarly, we used ROC curve analysis to clarify the relationship between AoPA and PAI (Figure 2B). The area under the curve of AoPA and PAI was 0.5667, the standard error was 0.104 , and the $95 \%$ CI was $0.363-0.770$. This model also indicated that AoPA had no significant predictive value for PAI $(p=0.5816)$. Thus, in our research, the predictive value of AoPA was bad.

\section{Predictive value of hPAI (pubic symphysis level) for PAI}

We then used ROC curve analysis to clarify the relationship between hPAI and PAI (Figure 2C). The area under the curve of hPAI and PAI was 0.940, the standard error was 0.037 , and the $95 \% \mathrm{CI}$ was $0.868-1.000$. This model indicated that hPAI had a significant predictive value for PAI $(p=0.0041)$. The boundary value of the prediction was $1.13 \mathrm{~cm}$, the sensitivity was 0.9 , and the specificity was 0.8667 . So, in our research, the predictive value of hPAI was good.

\section{Predictive value of AoPAI (pubic symphysis level) for PAI}

Next, we used ROC curve analysis to clarify the relationship between AoPAI and PAI (Figure 2D). The area under the curve of AoPAI and PAI was 0.95667, the standard error was 0.033, and the 95\% CI was 0.891-1.000. This model indicated that AoPAI had a significant predictive value for PAI $(p=0.0061)$. The boundary value was $26.32^{\circ}$, the sensitivity was 0.9 , and the specificity was 0.9 . Therefore, in our research, the predictive value of AoPAI was good.

\section{Predictive value of $h P A I^{M a x}$ for PAI}

Subsequently, we used ROC curve analysis to clarify the relationship between $\mathrm{hPAI}^{\mathrm{Max}}$ and PAI (Figure 2E). The area under the curve of $\mathrm{hPAI}^{\mathrm{Max}}$ and PAI was 0.87667 , the standard error was 0.077 , and the $95 \%$ CI was 0.725 1.000. This model indicated that $\mathrm{hPAI}^{\mathrm{Max}}$ had a significant predictive value for PAI $(p=0.0032)$. The boundary value of the prediction was $1.51 \mathrm{~cm}$, the sensitivity was 0.7 , and the specificity was 1 . Thus, in our research, the predictive value of hPAI ${ }^{\mathrm{Max}}$ was good.

\section{Predictive value of AoPAI ${ }^{\text {Max }}$ for PAI}

Similarly, we used ROC curve analysis to clarify the relationship between AoPAI ${ }^{\mathrm{Max}}$ and PAI (Figure 2F). The area under the curve of $\mathrm{hPAI}^{\mathrm{Max}}$ and PAI was 0.92667 , the standard error was 0.047 , and the $95 \%$ CI was $0.836-1.000$. This model indicated that AoPAI ${ }^{\mathrm{Max}}$ had a significant predictive value for PAI $(p=0.0072)$. The boundary value of the prediction was $28.37^{\circ}$, the sensitivity was 0.8 , and the specificity was 0.9333 . So, in our research, the predictive value of AoPAI ${ }^{\text {Max }}$ was good (see Table 2).

\section{Discussion}

Brachytherapy for prostate cancer is normally performed under the guidance of transrectal ultrasound. PAI is often a limiting factor for patients pursuing brachytherapy treatment. Pre-brachytherapy pubic arch evaluation is often performed by CT or TRUS. Using MRI to predict PAI is a new method [10].

Our study attempted to establish a simple and accurate method to predict PAI. To the best of our knowledge, this is the first study to systematically evaluate the predictive value of a variety of different parameters derived from MRI for pre-brachytherapy prediction of PAI.

If patients are in the head-low position (changing the angle of the ultrasound probe and the needle can have the same effect), the perineal puncture position moves down; therefore, PAI may be avoided to some degree. To overcome PAI, Tincher et al. [11] have reported that rotation of the pelvis from the supine to the lithotomy position, which can reduce PAI. Furthermore, directing the needle tip upward reduces PAI in the lithotomy position, when compared to the needle tip directed downward. The prin-

Table 2. Predicting PAl based on the specific parameters $(n=40)$

\begin{tabular}{lccccc} 
& $\begin{array}{c}\text { Areas under } \\
\text { the ROC }\end{array}$ & $\begin{array}{c}\text { Standard } \\
\text { error }\end{array}$ & $95 \% \mathrm{Cl}$ & $p$ & $\begin{array}{c}\text { Boundary } \\
\text { values }\end{array}$ \\
\hline PV & 0.592 & 0.110 & $0.376-0.807$ & 0.2975 & \\
\hline AoPA & 0.567 & 0.104 & $0.363-0.770$ & 0.5816 & $1.13 \mathrm{~cm}$ \\
\hline hPAl (pubic symphysis level) & 0.940 & 0.037 & $0.868-1.000$ & 0.0041 & $26.32^{\circ}$ \\
\hline AoPAl (pubic symphysis level) & 0.957 & 0.033 & $0.891-1.000$ & 0.0061 & $1.51 \mathrm{~cm}$ \\
\hline hPAI $^{\text {Max }}$ & 0.877 & 0.077 & $0.725-1.000$ & 0.0032 & $28.37^{\circ}$
\end{tabular}

PAI - pubic arch interference; PV - prostate volume; ROC - receiver operating characteristic; AoPA - the angle of the pubic arch; hPAl - the height of PAl; AoPAl - the angle of PAl; $h P A M^{M a x}$ - the maximum height of PAl; AoPAlMax - the maximum angle of PAI 
ciple of PAI analyzed by Tincher is similar to that of our research.

As the ultrasound probe is placed in the rectum, the angle of the head-low position cannot be too much. Moreover, if the angle of the head-low position is too large, the ultrasound probe cannot be close to the prostate, leading to unclear prostate imaging in the ultrasound examination. According to the principle of geometry, the angle of the posterior edge of the pubic and the anterior edge of prostate seems to be the key factor of PAI; however, this idea has not been systemically tested nor has any reliable predictor of PAI been reported.

In our research, we used six new parameters derived from MRI, including PV, AoPA, AoPAI (pubic symphysis level), hPAI (pubic symphysis level), AoPAI ${ }^{\text {Max }}$, and $\mathrm{hPAI}^{\mathrm{Max}}$; we found that the AoPAI, hPAI, hPAI ${ }^{\mathrm{Max}}$, and AoPAI ${ }^{\mathrm{Max}}$ could predict PAI. Among all of these parameters that have a predictive value, it is worth noting the following: 1 . The parameters from the pubic symphysis level could be measured more conveniently than from any other level and importantly, at this level the MRI results are clearer; therefore, we recommend using the images from the pubic symphysis level; 2 . The angle is the key factor of PAI, and it is easily and accurately measured as well as easily standardized, since it is difficult to confirm the highest point of the anterior prostate, and the scale used to measure the height might be different between different hospitals. Together, we argue that AoPAI could be used as a more reliable and convenient parameter to predict PAI.

The prostate ligament makes the prostate and pubic position relatively fixed. We have studied six parameters both in lithotomy and supine MRI positions, and we found that lithotomy and supine positions in MRI did not make difference, but the supine position is more convenient for clinical application.

However, the limitations of this study include the following: This was a retrospective study with relatively few cases, i.e., only 10 patients with PAI were included. In order to ensure the therapeutic effect of treatment, we only selected patients without PAI according to TRUS, $\mathrm{CT}$, and MRI, based on our previous experience. The selection bias of the inclusion criteria may affect the accuracy of the results. And, we only selected patients with PV $>40 \mathrm{ml}$. Therefore, future, large scale, and prospective studies are warranted to confirm this finding, and to obtain more accurate predictive boundary values.

In conclusion, this study provides novel parameters to predict PAI, i.e., AoPAI, hPAI, hPAI ${ }^{\mathrm{Max}}$, and AoPAI ${ }^{\mathrm{Max}}$. Furthermore, because the plane of pubic symphysis can be clearly displayed on nuclear magnetic resonance, measuring angle is more convenient and accurate than measuring height. We suggest that AoPAI (pubic symphysis level) is the most convenient and accurate parameter for predicting PAI.

\section{Disclosure}

Authors report no conflict of interest.

\section{References}

1. Taira AV, Merrick GS, Butler WM et al. Long-term outcome for clinically localized prostate cancer treated with permanent interstitial brachytherapy. Int J Radiat Oncol Biol Phys 2011; 79: 1336-1342.

2. Hayashi N, Izumi K, Sano F et al. Ten-year outcomes of I-125 low-dose-rate brachytherapy for clinically localized prostate cancer: a single-institution experience in Japan. World J Urol 2015; 33: 1519-1526.

3. Stish BJ, Davis BJ, Mynderse LA et al. Brachytherapy in the management of prostate cancer. Surg Oncol Clin N Am 2017; 26: 491-513

4. Bellon J, Wallner K, Ellis W et al. Use of pelvic CT scanning to evaluate pubic arch interference of transperineal prostate brachytherapy. Int J Radiat Oncol Biol Phys 1999; 43: 579-581.

5. Nguyen PL, Alibhai SM, Basaria S et al. Adverse effects of androgen deprivation therapy and strategies to mitigate them. Eur Urol 2015; 67: 825-836.

6. Henderson A, Laing RW, Langley SE. Identification of pubic arch interference in prostate brachytherapy: Simplifying the transrectal ultrasound technique. Brachytherapy 2003; 2: 240245 .

7. Fukada J, Shigematsu N, Nakashima J et al. Predicting pubic arch interference in prostate brachytherapy on transrectal ultrasonography-computed tomography fusion images. J Radiat Res 2012; 53: 753-759.

8. Davis BJ, Horwitz EM, Lee WR et al. American Brachytherapy Society consensus guidelines for transrectal ultrasound-guided permanent prostate brachytherapy. Brachytherapy 2012; 11: 6-19.

9. Ryu B, Bax J, Edirisinge C et al. Prostate brachytherapy with oblique needles to treat large glands and overcome pubic arch interference. Int J Radiat Oncol Biol Phys 2012; 83: 14631472.

10. Blanchard P, Menard C, Frank SJ et al. Clinical use of magnetic resonance imaging across the prostate brachytherapy workflow. Brachytherapy 2017; 16: 728-733.

11. Tincher SA, Kim RY, Ezekiel MP et al. Effects of pelvic rotation and needle angle on pubic arch interference during transperineal prostate implants. Int J Radiat Oncol Biol Phys 2000; $47: 361-363$ 